\title{
GENERALIZED BOMBER AND FIGHTER PROBLEMS: OFFLINE OPTIMAL ALLOCATION OF A DISCRETE ASSET
}

\author{
ABBA M. KRIEGER, * Wharton School of the University of Pennsylvania \\ ESTER SAMUEL-CAHN, ${ }^{* *}$ The Hebrew University of Jerusalem
}

\begin{abstract}
The classical bomber problem concerns properties of the optimal allocation policy of a given number, $n$, of anti-aircraft missiles, with which an airplane is equipped. The airplane begins at a distance $t>0$ from its destination and uses some of the anti-aircraft missiles when intercepted by enemy planes that appear according to a homogeneous Poisson process. The goal is to maximize the probability of reaching its destination. The fighter problem deals with a similar situation, but the goal is to shoot down as many enemy planes as possible. The optimal allocation policies are dynamic, depending upon both the number of missiles and the time which remains to reach the destination when the enemy is met. The present paper generalizes these problems by allowing the number of enemy planes to have any distribution, not just Poisson. This implies that the optimal strategies can no longer be dynamic, and are, in our terminology, offline. We show that properties similar to those holding for the classical problems hold also in the present case. Whether certain properties hold that remain open questions in the dynamic version are resolved in the offline version. Since 'time' is no longer a meaningful way to parametrize the distributions for the number of encounters, other more general orderings of distributions are needed. Numerical comparisons between the dynamic and offline approaches are given.
\end{abstract}

Keywords: Dynamic allocation; invincible fighter; frail fighter; hazard rate dominance; likelihood ratio dominance; optimality properties

2010 Mathematics Subject Classification: Primary 62L99

Secondary $60 \mathrm{~K} 10$

\section{Introduction}

The classical bomber problem, as first considered in [4] and [5], is described as follows. An airplane equipped with a known number $n$ of anti-aircraft missiles and at a known distance $t>0$ from its destination is intercepted by enemy aircrafts, appearing according to a homogeneous Poisson distribution with intensity 1 . Its goal is to allocate its $n$ missiles optimally, so as to maximize the probability of reaching its destination. The fighter problem (see [2]) shares the same probabilistic structure, but the goal is changed to that of maximizing the expected total number of enemy planes shot down. The original assumption was that each missile independently hits the enemy with probability $1-q$, where $0<q<1$. If the enemy was not

Received 13 September 2012; revision received 11 October 2012.

* Postal address: Department of Statistics, Wharton School of the University of Pennsylvania, Philadelphia, PA 19104, USA. Email address: krieger@wharton.upenn.edu

** Postal address: Department of Statistics and Center for Rationality, The Hebrew University of Jerusalem, Jerusalem 91905, Israel. Email address: scahn@mscc.huji.ac.il 
hit, it destroys the airplane with probability $1-u$, where $0 \leq u \leq 1$ for the fighter and $u>0$ for the bomber.

Denote the optimal number of missiles to allocate when in state $(n, t)$ by $K(n, t)$. Three conjectures have been postulated regarding the properties of $K(n, t)$.
A. For fixed $n, K(n, t)$ is nonincreasing in $t$.
B. For fixed $t, K(n, t)$ is nondecreasing in $n$.
C. For fixed $t, n-K(n, t)$ is nondecreasing in $n$.

For the above description the findings are: for the bomber, $\mathrm{A}$ and $\mathrm{C}$ hold and $\mathrm{B}$ is still unsettled; for the fighter, $\mathrm{C}$ holds, $\mathrm{B}$ fails, and $\mathrm{A}$ is still unsettled, except when $u=0$ and $u=1$, where it holds. The assumptions about the hitting and survival probabilities can be generalized, as we describe below. In the present paper we generalize the above fighter problem in Section 3 and bomber problem in Section 4. Instead of restricting the total number of enemy planes met to have a Poisson distribution with mean $t$, we let the total number, $X$, have any known, nonnegative integer-valued distribution. Thus, there is no longer any time element involved. This implies that it is no longer meaningful to consider online, i.e. dynamic, allocations. The allocation of the missiles can and must therefore be made ahead, depending on $X$ and $n$. An allocation is denoted $J_{n}=\left(j_{1}, \cdots, j_{n}\right)$ with $j_{k} \geq 0$ and $\sum_{k=1}^{n} j_{k}=n$, where $j_{k}$ denotes the number of missiles to be used at the $k$ th potential encounter. Let $J^{*}(X, n)$ denote an optimal allocation in situation $(X, n)$. Conjectures $\mathrm{B}$ and $\mathrm{C}$ can be restated as follows.

Conjecture B. For any $X, j_{1}^{*}(X, n)$ is nondecreasing in $n$.

Conjecture C. For any $X, n-j_{1}^{*}(X, n)$ is nondecreasing in $n$.

But, since there is no longer any time element involved, we need another way of comparing the distributions of two random variables, $X$ and $Y$. It turns out that the needed order concept for the fighter problem is the hazard rate (hr) order, while, for the bomber, it is the likelihood ratio (lr) order, both concepts being explained in detail later. The main results in the present paper are the generalized equivalents of $\mathrm{A}$.

Conjecture A. (For the fighter.) If $Y<_{\mathrm{hr}} X$ then $j_{1}^{*}(Y, n) \geq j_{1}^{*}(X, n)$.

Conjecture A. (For the bomber.) If $Y<\operatorname{lr} X$ then $j_{1}^{*}(Y, n) \geq j_{1}^{*}(X, n)$.

We generalize this and show that, under the above conditions, the entire $J^{*}(Y, n)$ vector majorizes the $J^{*}(X, n)$ vector. Furthermore, we show that Conjecture $\mathrm{B}$ is false both for the bomber and the fighter, while Conjecture $\mathrm{C}$ holds for both. These results hold under more general conditions than those given above. Let $a(j)$ denote the hitting probability when $j$ missiles are used, and let $c(j)$ denote the corresponding survival probability, $j=1,2, \ldots$ Note that, for the bomber problem, only the $c(j)$ sequence plays a role, and a sufficient condition for the above results to hold is that $c(j)$ be a nondecreasing log-concave sequence. For the fighter problem, we need in addition that the $a(j)$ sequence be nondecreasing and concave. We conclude with a comparison in Section 5 of the offline and online situations when $X$ follows a Poisson process and, hence, such a comparison is possible. Even though the online situation necessarily yields higher values of the objective, the difference is shown to be quite small in the cases we consider, particularly for the fighter problem. 


\section{Formulation and notation}

There are two aspects to the dynamics of the problem. The first issue is the way in which the enemy is shot down and, relatedly, the way in which the bomber or fighter survives. The second is the random variable, $X$, that describes the number of encounters.

Let

$$
a(j)=\mathbb{P}(\text { hitting, when } j \text { missiles are used })
$$

and

$$
c(j)=\mathbb{P} \text { (surviving, when } j \text { missiles are used). }
$$

We assume that

1. $a(0)=0<a(1)<a(2)<\cdots$,

2. $c(0) \leq c(1) \leq c(2) \leq \cdots$.

For many of the results we make the additional assumptions that $a(j)$ is concave, that is, $a(j+1)-a(j)$ is nonincreasing in $j$ for $j \geq 1$, and $c(j)$ is log-concave, that is, $c(j+1) / c(j)$ is nonincreasing in $j$. Note that log-concavity is a weaker condition than concavity. To avoid trivialities, we assume for the bomber problem that $c(0)=0$ and the $c$-sequence is strictly increasing.

In the classical model in the literature it is assumed that $a(j)=1-q^{j}$ and $c(j)=a(j)+$ $(1-a(j)) u$ for $j \geq 1$, where $0<q<1$ and $0 \leq u \leq 1$. This model assumes that the missiles act independently, each hitting with probability $1-q$, and if the enemy is not hit, he destroys you with fixed (known) probability $1-u$. For ease of reference later, we refer to this model by $M(q, u)$. For the fighter problem when $u=0$ and $u=1$, the problems are referred to as the 'frail' fighter and 'invincible' fighter, respectively.

The second aspect is the behavior of the number of encounters. In the online version of the problem, it is necessary to model the way in which encounters occur in time. In the literature, the problem that allows for allocation decisions to be made sequentially at the time an encounter occurs is often referred to as the 'dynamic' problem. We use the terminology online instead of dynamic to contrast it to the offline version considered here. Typically, two time models have been considered in the literature.

1. Continuous time, where the assumption is that the arrival of the enemy planes is according to a homogeneous Poisson process, where homogenity is assumed for convenience only (see [4] and [5]). In the offline situation, when we use the notation $M(q, u, t)$, we are referring to $X$ being Poisson with mean $t$ and the model being $M(q, u)$.

2. Discrete time, where at each instant $t$ an enemy plane appears with probability $p$, or, more generally, with probability $p_{t}$ (see [8]).

These references discuss the bomber problem only, but later references discuss also the fighter problem for these two models. The important feature of these models is that they have independent increments, which plays an essential role in the online case, but cannot easily be exploited when studying the offline case, where the nature of 'time' is lost.

The classical conjecture, Conjecture A, that we want to prove for the offline version is that, for a fixed $n$, as the number of encounters tends to increase, it is best to use fewer missiles initially. In the online version, this translates to: for a fixed number of missiles, $n$, the more time that one has at his disposal, the fewer missiles one should spend at the present enemy plane. This conjecture applies to both the fighter and bomber problems. 
In order to specify the offline version, we need to describe what we mean by the number of encounters 'tends to increase', that is, $Y<X$. Among the various order relations that exist between random variables $X$ and $Y$ in the literature, we will consider three. See [6] for an excellent exposition. The weakest form is stochastic dominance, which is denoted by $Y<_{\text {st }} X$. This requires that $\mathbb{P}(X \geq x) \geq \mathbb{P}(Y \geq x)$ for all $x$, with strict inequality for at least one $x$. The next ordering is the hazard rate dominance, which is defined as follows.

Definition 1. For discrete random variables $X$ and $Y, Y$ is hazard rate smaller than $X$, denoted by $Y<_{\mathrm{hr}} X$, if

$$
\frac{\mathbb{P}(Y=x)}{\mathbb{P}(Y \geq x)} \geq \frac{\mathbb{P}(X=x)}{\mathbb{P}(X \geq x)},
$$

with strict inequality for at least one $x$.

This is Equation (1.B.7) of [6]. It follows that $Y<_{\mathrm{hr}} X$ is equivalent to

$$
\frac{\mathbb{P}(X \geq j)}{\mathbb{P}(Y \geq j)} \geq \frac{\mathbb{P}(X \geq k)}{\mathbb{P}(Y \geq k)}
$$

for all $0 \leq k<j$. Equation (1) can be rewritten as

$$
\frac{\mathbb{P}(X \geq j)}{\mathbb{P}(X \geq k)} \geq \frac{\mathbb{P}(Y \geq j)}{\mathbb{P}(Y \geq k)} .
$$

It is also shown in the above reference that the hazard rate order is weaker than the likelihood ratio order. The latter, $Y<_{\operatorname{lr}} X$, is defined by the condition that $\mathbb{P}(X=j) / \mathbb{P}(Y=j)$ is a nondecreasing function of $j$.

It turns out that the hazard rate order is needed to prove our main result for the fighter problem, while the likelihood ratio order is needed to prove a similar result for the bomber problem. It is immediate that if $X \sim \operatorname{Poisson}\left(\mu_{1}\right)$ and $Y \sim \operatorname{Poisson}\left(\mu_{2}\right)$ with $\mu_{1}>\mu_{2}$, then $X>_{\operatorname{lr}} Y$. Similarly, if $X \sim \operatorname{Binomial}\left(n_{1}, p\right)$ and $Y \sim \operatorname{Binomial}\left(n_{2}, p\right)$ with $n_{1}>n_{2}$, then $X>_{\operatorname{lr}} Y$. Also, if $X \sim \operatorname{Geometric}\left(p_{1}\right)$ and $Y \sim \operatorname{Geometric}\left(p_{2}\right)$ with $p_{1}<p_{2}$, then $X>_{\operatorname{lr}} Y$. Many other parametric examples can be given.

In order to prove Conjecture A, we 'connect' $X$ to $Y$ through $X_{\alpha}$, which is defined by

$$
\mathbb{P}\left(X_{\alpha} \geq k\right)=\alpha \mathbb{P}(X \geq k)+(1-\alpha) \mathbb{P}(Y \geq k)
$$

for $0 \leq \alpha \leq 1$. We first prove the following result about $X_{\alpha}$.

Lemma 1. Let $Y<_{\mathrm{hr}} X(Y<\operatorname{lr} X)$. Then, for $0 \leq \alpha^{\prime}<\alpha \leq 1, X_{\alpha^{\prime}}<_{\mathrm{hr}} X_{\alpha}\left(X_{\alpha^{\prime}}<\operatorname{lr} X_{\alpha}\right)$.

Proof. For the hazard rate, it suffices to show that

$$
\frac{\mathbb{P}\left(X_{\alpha} \geq j\right)}{\mathbb{P}\left(X_{\alpha} \geq k\right)}=\frac{\alpha \mathbb{P}(X \geq j)+(1-\alpha) \mathbb{P}(Y \geq j)}{\alpha \mathbb{P}(X \geq k)+(1-\alpha) \mathbb{P}(Y \geq k)}
$$

is increasing in $\alpha$ for $j>k$. If we take the derivative of the right-hand side of the above expression with respect to $\alpha$, its numerator is easily seen to be

$$
\mathbb{P}(X \geq j)(\mathbb{P}(Y \geq k)-\mathbb{P}(Y \geq j) \mathbb{P}(X \geq k)) .
$$

This expression is nonnegative from (2). The result for the likelihood ratio proceeds in the same way by considering $\mathbb{P}\left(X_{\alpha}=j\right) / \mathbb{P}\left(X_{\alpha}=k\right)$ for $j>k$. 
Allocation policies are of the form $J_{n}=\left(j_{1}, \ldots, j_{n}\right)$, where $j_{i}$ denotes the amount of the total $n$ missiles to be allocated to the $i$ th encounter if such an encounter takes place. Hence, the $j_{i}$ are nonnegative and integer-valued with $\sum_{i=1}^{n} j_{i}=n$. We denote the probability of surviving through $k$ encounters, using $J_{n}$, by

$$
s_{k}\left(J_{n}\right)=\prod_{i=1}^{k} c\left(j_{i}\right)
$$

with $s_{0}\left(J_{n}\right)=1$. Hence, the objective function in the fighter problem, i.e. the expected value of the number of enemy planes that are knocked down, can be written as

$$
F\left(X, J_{n}\right)=\sum_{k=1}^{n} \mathbb{P}(X \geq k) s_{k-1}\left(J_{n}\right) a\left(j_{k}\right) .
$$

Similarly, the objective function in the bomber problem, the probability of making it home, can be written as

$$
B\left(X, J_{n}\right)=\sum_{k=0}^{n} \mathbb{P}(X=k) s_{k}\left(J_{n}\right) .
$$

In proving Conjecture A, the stochastic order does not suffice. We need the stronger hazard rate order for the fighter problem and likelihood ratio order for the bomber problem. But in terms of the behavior of the objective functions, the stochastic order suffices. Specifically, for any sequences $0<a(j) \leq 1$ and $0<c(j) \leq 1$, if $Y<_{\text {st }} X$ then, for all $J_{n}$, we have $F\left(Y, J_{n}\right) \leq F\left(X, J_{n}\right)$ and $B\left(Y, J_{n}\right) \geq B\left(X, J_{n}\right)$. For the fighter problem, this is immediate from (4). For the bomber problem, note that (5) can be written as

$$
B\left(X, J_{n}\right)=1-\sum_{k=1}^{n+1} \mathbb{P}(X \geq k)\left(1-c\left(j_{k}\right)\right) s_{k-1}\left(J_{n}\right) .
$$

We omit the $X$ in the above notation, when the distribution of $X$ is fixed. Also, note that the objective function in the bomber problem depends only on the distribution of $X$ and the $c(j)$ sequence, while the objective function in the fighter problem depends on the distribution of $X$ as well as both the $c(j)$ and the $a(j)$ sequences.

We denote the optimal strategy by $J_{n}^{*}=\left(j_{1}^{*}, \ldots, j_{n}^{*}\right)$ (for each problem separately). In general, $j_{k}^{*}=j_{k}^{*}(X, n)$ depends on the distribution of the number of encounters, $X$, and on the number of missiles that are available at the outset, $n$. When $X$ and/or $n$ are fixed for a particular result, we omit the dependence of $j_{k}^{*}$ on these arguments to simplify the notation.

Convention. Suppose that $\hat{J}_{n}=\left(\hat{j}_{1}, \ldots, \hat{j_{n}}\right)$ and $\tilde{J}_{n}=\left(\tilde{j}_{1}, \ldots, \tilde{j}_{n}\right)$ are both optimal. Then $J_{n}^{*}=\left(j_{1}^{*}, \ldots, j_{n}^{*}\right)$ will be chosen so that $j_{1}^{*}$ is minimal.

It is easy to verify that $j_{1}^{*} \geq j_{2}^{*} \geq \cdots \geq j_{n}^{*}$ for both the fighter and bomber problems by a simple interchange argument.

Conjectures $\mathrm{A}, \mathrm{B}$, and $\mathrm{C}$ have received much attention in the online situation. The purpose of the next two sections is to prove or find counterexamples to these three conjectures in the offline situation. Before stating the conjectures for the offline situation we introduce the sense in which one allocation favors using more missiles at earlier encounters than another allocation. 
Definition 2. $J=\left(j_{1}, \ldots, j_{n}\right)$ majorizes $J^{\prime}=\left(j_{1}^{\prime}, \ldots, j_{n}^{\prime}\right)$ if $\sum_{i=1}^{k} j_{i} \geq \sum_{i=1}^{k} j_{i}^{\prime}$ for all $1 \leq k \leq n$ with equality when $k=n$. If there is at least one $k$ for which the inequality is strict then $J$ strictly majorizes $J^{\prime}$.

The three conjectures can be stated as follows.

Conjecture A. If $X>_{h r} Y\left(X>_{1 \mathrm{r}} Y\right)$ then $j_{1}^{*}(X) \leq j_{1}^{*}(Y)$ for the fighter problem (bomber problem). We extend this to $J^{*}(Y)$ majorizes $J^{*}(X)$.

Conjecture B. For any $X$, if $n_{1}>n_{2}$ then $j_{1}^{*}\left(n_{1}\right) \geq j_{1}^{*}\left(n_{2}\right)$.

Conjecture C. For any $X$, if $n_{1}>n_{2}$ then $n_{1}-j_{1}^{*}\left(n_{1}\right) \geq n_{2}-j_{1}^{*}\left(n_{2}\right)$.

Conjecture A implies that if one is likely to face more encounters then fewer of the $n$ missiles should be used on the first encounter. In Conjectures B and C, we assume that the distribution for the number of encounters is fixed. Conjecture B implies that the more missiles one has, the more missiles one should tend to use on the first encounter. Conjecture $\mathrm{C}$ implies that the more missiles at one's disposal, the more missiles should be held back for possible future encounters.

\section{The fighter problem}

The fighter problem was first addressed by Weber [9]. He considered the online problem for discrete time, with $M(0.5,0)$, and showed that B fails, since $j_{1}^{*}(10)=4$ while $j_{1}^{*}(11)=3$. Shepp et al. [7] considered the frail fighter situation, where the enemy has an infinite number of planes and ammunition is continuous. Since there is no 'time' involved, this is in essence an offline problem. One main result is that Conjecture B fails for this situation. The fighter terminology was introduced by Bartroff et al. [3]. They also considered continuous ammunition. Bartroff and Samuel-Cahn [2] considered continuous time with $M(0.5,0)$, and showed that Conjecture B fails for all $t>2.694 \ldots$, and holds for all smaller values of $t$. In this section we consider the above three conjectures in the context of the fighter problem. The only case where Conjecture $\mathrm{B}$ holds for the online fighter problem is the invincible case, where $u=1$. Below we show that, for the offline fighter problem, it also holds in that case.

Theorem 1. Let $c(j) \equiv 1$, and let the a $(j)$ sequence be concave. Then, for any $X$, Conjecture $B$ holds for the offline problem, i.e. $j_{1}^{*}(n) \leq j_{1}^{*}(m)$ for all $n<m$.

Proof. If $\mathbb{P}(X>1)=0$, the theorem is trivially true. Assume that $\mathbb{P}(X>1)>0$ and the theorem is false. Then there exists an $n$ with $j_{1}^{*}(n)>j_{1}^{*}(n+1)$. This implies that there must exist a $k \leq n$ with $\mathbb{P}(X \geq k)>0$ and with $j_{k}^{*}(n)<j_{k}^{*}(n+1)$. Because of the optimality of $J^{*}(n)$, it follows that if we reduce the allocation for the first encounter by one and increase the allocation to the $k$ th encounter by one, we must do worse. This implies that

$$
\begin{aligned}
\mathbb{P}(X \geq 1) a\left(j_{1}^{*}(n)\right)+\mathbb{P}(X \geq k) a\left(j_{k}^{*}(n)\right) \\
\quad>\mathbb{P}(X \geq 1) a\left(j_{1}^{*}(n)-1\right)+\mathbb{P}(X \geq k) a\left(j_{k}^{*}(n)+1\right) .
\end{aligned}
$$

Strict inequality in (6) follows from the convention that the optimal solution in the case of ties goes to the one with smaller first entry. It follows from (6) that

$$
\frac{\mathbb{P}(X \geq 1)}{\mathbb{P}(X \geq k)}>\frac{a\left(j_{k}^{*}(n)+1\right)-a\left(j_{k}^{*}(n)\right)}{a\left(j_{1}^{*}(n)\right)-a\left(j_{1}^{*}(n)-1\right)} .
$$


TABLE 1: Expected number of enemy planes knocked down for $M(0.5,0,5)$.

\begin{tabular}{|c|c|c|c|c|c|c|c|c|c|c|}
\hline \multicolumn{5}{|c|}{ Four missiles } & \multicolumn{6}{|c|}{ Five missiles } \\
\hline$j_{1}$ & $j_{2}$ & $j_{3}$ & $j_{4}$ & $F(X, 4)$ & $j_{1}$ & $j_{2}$ & $j_{3}$ & $j_{4}$ & $j_{5}$ & $F(X, 5)$ \\
\hline 4 & 0 & 0 & 0 & 0.9312 & 5 & 0 & 0 & 0 & 0 & 0.9622 \\
\hline 3 & 1 & 0 & 0 & 1.2889 & 4 & 1 & 0 & 0 & 0 & 1.3810 \\
\hline 2 & 2 & 0 & 0 & 1.2847 & 3 & 2 & 0 & 0 & 0 & 1.4988 \\
\hline 2 & 1 & 1 & 0 & 1.2689 & 3 & 1 & 1 & 0 & 0 & 1.4804 \\
\hline \multirow[t]{3}{*}{1} & 1 & 1 & 1 & 0.8919 & 2 & 2 & 1 & 0 & 0 & 1.5309 \\
\hline & & & & & 2 & 1 & 1 & 1 & 0 & 1.3378 \\
\hline & & & & & 1 & 1 & 1 & 1 & 1 & 0.9094 \\
\hline
\end{tabular}

Similarly, because of the optimality of $J^{*}(n+1)$, it follows that if we increase the allocation of the first encounter by one and reduce the allocation of the $k$ th encounter by one we must do no better. Hence,

$$
\begin{aligned}
\mathbb{P}(X \geq 1) a\left(j_{1}^{*}(n+1)\right)+\mathbb{P}(X \geq k) a\left(j_{k}^{*}(n+1)\right) \\
\quad \geq \mathbb{P}(X \geq 1) a\left(j_{1}^{*}(n+1)+1\right)+\mathbb{P}(X \geq k) a\left(j_{k}^{*}(n+1)-1\right) .
\end{aligned}
$$

It follows from (8) that

$$
\frac{\mathbb{P}(X \geq 1)}{\mathbb{P}(X \geq k)} \leq \frac{a\left(j_{k}^{*}(n+1)\right)-a\left(j_{k}^{*}(n+1)-1\right)}{a\left(j_{1}^{*}(n+1)+1\right)-a\left(j_{1}^{*}(n+1)\right)} .
$$

Under the assumption that $j_{k}^{*}(n)+1 \leq j_{k}^{*}(n+1)$, it follows from the concavity of the $a(j)$ sequence that the numerator in (7) is at least as large as the numerator in (9) and the denominator in (9) is at least as large as the denominator in (7). Hence, the right-hand side of (7) is at least as large as the right-hand side of (9), which is a contradiction.

Assertion 1. For the offline fighter problem, Conjecture B is false for an $M(q, u, t)$ model.

Specifically, we consider the $M(0.5,0,5)$ model, which was considered in the online case. Table 1 provides the values for the expected number of enemy planes that are shot down when $n=4$ and $n=5$ missiles are available for all monotone vectors $J_{n}$. It is apparent from Table 1 that $J_{4}^{*}=(3,1,0,0)$ and $J_{5}^{*}=(2,2,1,0,0)$. Hence, $j_{1}^{*}(4)=3>j_{1}^{*}(5)=2$.

We now show that Conjecture $\mathrm{C}$ holds for the fighter problem. Before stating and proving the theorem, note that

$$
F\left(X, J_{n}^{*}\right)=\mathbb{P}(X \geq 1)\left[a\left(j_{1}^{*}\right)+c\left(j_{1}^{*}\right) F\left(X^{\prime}, J_{n-j_{1}^{*}}^{*}\right)\right]
$$

where $X^{\prime}$ has conditional distribution of $X-1$ given $X \geq 1$, and $j_{i}{ }^{*}=j_{i+1}^{*}, i=1, \ldots, n-1$. This can be written as

$$
F\left(X, J_{n}^{*}\right)=\mathbb{P}(X \geq 1)\left[a\left(j_{1}^{*}\right)+c\left(j_{1}^{*}\right) R_{F}\left(X^{\prime}, n-j_{1}^{*}\right)\right]
$$

(where $R$ stands for the 'remainder').

Theorem 2. Assume that the $a(j)$ sequence is concave and nondecreasing and that the $c(j)$ sequence is log-concave and nondecreasing. Then Conjecture C holds for the offline fighter problem. 
Proof. We want to show that $n-j_{1}^{*}(n) \leq n+1-j_{1}^{*}(n+1)$. Our proof is by contradiction. Thus, assume that

$$
j_{1}^{*}(n+1)>j_{1}^{*}(n)+1 .
$$

For ease of notation, let $j=j_{1}^{*}(n)$ and $h=j_{1}^{*}(n+1)$. Since $j$ is optimal for $n$, it is preferred to $h-1$ for $n$; thus, using (10), $a(j)+c(j) R_{F}\left(X^{\prime}, n-j\right) \geq a(h-1)+c(h-1) R_{F}\left(X^{\prime}, n+1-h\right)$. Hence,

$$
R_{F}\left(X^{\prime}, n+1-h\right) \leq \frac{a(j)-a(h-1)}{c(h-1)}+\frac{c(j)}{c(h-1)} R_{F}\left(X^{\prime}, n-j\right) .
$$

Similarly, since $h$ is optimal for $n+1$, it is preferred to $j+1$ for $n+1$; thus,

$$
a(h)+c(h) R_{F}\left(X^{\prime}, n+1-h\right)>a(j+1)+c(j+1) R_{F}\left(X^{\prime}, n-j\right) .
$$

Strict inequality holds by the convention that in the case of ties the smaller number of configurations is preferred and $h>j+1$. Hence,

$$
R_{F}\left(X^{\prime}, n+1-h\right)>\frac{a(j+1)-a(h)}{c(h)}+\frac{c(j+1)}{c(h)} R_{F}\left(X^{\prime}, n-j\right) .
$$

Again, the inequality is strict from (11) and the strict monotonicity of the $a$ sequence. Now our assumptions about the $a(j)$ and $c(j)$ sequences imply, using (11), that

$$
\frac{a(j+1)-a(h)}{c(h)} \geq \frac{a(j)-a(h-1)}{c(h-1)}
$$

and

$$
\frac{c(j+1)}{c(h)} \geq \frac{c(j)}{c(h-1)} .
$$

Clearly, (12) and (13) contradict each other and, thus, (11) cannot hold.

We now turn to proving Conjecture A for the offline fighter problem. The analogue in the online version is still an open problem. Since $n$ is fixed, we do not include $n$ in the notation that follows.

We first consider a series of lemmas, the proofs of which appear in Appendix A.

Let $\hat{J}(g, Y)$ be the allocation that uses $g$ missiles on the first encounter (provided there is one) and allocates the remaining $n-g$ missiles optimally for $Y$ for the remaining potential encounters.

Lemma 2. If $g<h$ then $\hat{J}(h, X)$ majorizes $\hat{J}(g, X)$.

Denote the contribution to $F$ from the $k$ th encounter, using $J$, assuming this encounter takes place, by $v_{k}(J)=s_{k-1}(J) a\left(j_{k}\right)$. Also, let $m(J)=\operatorname{argmax}_{k}\left\{v_{k}(J)>0\right\}$.

Lemma 3. If $J=\hat{J}(h, X)$ and $J^{\prime}=\hat{J}(g, X)$ with $g<h$, then $v_{m\left(J^{\prime}\right)}(J) \leq v_{m\left(J^{\prime}\right)}\left(J^{\prime}\right)$.

Let $V=\left(v_{1}, \ldots, v_{n}\right)$ and similarly for $V^{\prime}$. We now turn to the main lemma.

Lemma 4. Let $F(X, V)=\sum_{k=1}^{n} \mathbb{P}(X \geq k) v_{k}$. If $v_{1} \geq v_{1}^{\prime}, v_{n} \leq v_{n}^{\prime}$, and $F(Y, V) \leq F\left(Y, V^{\prime}\right)$, then, for any $X$ such that $Y<_{\mathrm{hr}} X, F(X, V) \leq F\left(X, V^{\prime}\right)$. 
We apply these lemmas with $J=\hat{J}(h, Y), J^{\prime}=\hat{J}(g, Y)$, and $g<h$. By virtue of Lemma 2 and Lemma 3, the conditions of Lemma 4 are established with $v_{k}=v_{k}(\hat{J}(h, Y))$ and $v_{k}^{\prime}=v_{k}^{\prime}(\hat{J}(g, Y))$ for $k=1, \ldots, n$. Hence, if $\hat{J}(g, Y)$ has at least as high an objective as $\hat{J}(h, Y)$ when $Y$ governs the number of encounters, it will also have at least as high an objective when $X$ governs the number of encounters for any $X$ such that $Y<\mathrm{hr} X$.

Theorem 3. If $Y<\mathrm{hr} X$ then $j_{1}^{*}(Y) \geq j_{1}^{*}(X)$.

Proof. If $\mathbb{P}(Y>1)=0$, the result is obvious. Thus, assume that $\mathbb{P}(Y>1)>0$. The proof follows by contradiction. Assume that there exists $Y<_{\mathrm{hr}} X$ with $g=j_{1}^{*}(Y)<h=j_{1}^{*}(X)$. First we connect $Y$ to $X$ through $X_{\alpha}$ using (3). Note that, for a fixed allocation $J^{\prime \prime}, F\left(X_{\alpha}, J^{\prime \prime}\right)$ is a linear function in $\alpha$ from (3). This implies that $F(g, \alpha):=F\left(X_{\alpha}, \hat{J}\left(g, X_{\alpha}\right)\right)$ is an increasing, continuous, piecewise-linear function in $\alpha$. If two different allocations have the same objective at a given $\alpha$, the allocation that is chosen at that $\alpha$ is the one that goes from below to above. We define $F(h, \alpha)$ similarly. Hence, when $F(g, \alpha)$ and $F(h, \alpha)$ cross, either $F(g, \alpha)$ goes from below to above or vice versa. Also, the two piecewise-linear functions cross a finite number of times as there is a finite number of possible allocations.

By assumption, $F(g, 0) \geq F(h, 0)$ since $g=j_{1}^{*}(Y)$, while $F(g, 1)<F(h, 1)$ as $h=$ $j_{1}^{*}(X)$. By continuity of the $F(g, \alpha)$ and $F(h, \alpha)$ curves, these must therefore intersect. Hence, there must exist at least one $\alpha$ at which $F(g, \alpha)=F(h, \alpha)$. Let $\alpha_{e}$ be the supremum of the finite number of such $\alpha$ where $F(g, \alpha)$ and $F(h, \alpha)$ cross. There is an $\alpha_{m}>\alpha_{e}$ such that $\hat{J}\left(g, X_{\alpha_{m}}\right)=\hat{J}\left(g, X_{\alpha_{e}}\right)$ and $\hat{J}\left(h, X_{\alpha_{m}}\right)=\hat{J}\left(h, X_{\alpha_{e}}\right)$. It follows that $F\left(g, \alpha_{m}\right)<F\left(h, \alpha_{m}\right)$ from the assumption that $F(g, 1)<F(h, 1)$. This contradicts Lemma 4 as $X_{\alpha_{e}}<_{\mathrm{hr}} X_{\alpha_{m}}$.

We extend Theorem 3 as follows.

Corollary 1. If $Y<{ }_{\mathrm{hr}} X$ then $J^{*}(Y)$ majorizes $J^{*}(X)$.

Proof. The result follows by induction on the number of missiles, $n$. It is clearly true for $n=1$ as there is only one possible allocation. Assume that it is true if the number of missiles is $n$ or fewer. Consider $n+1$ missiles. Let

$$
g=j_{1}^{*}(X, n+1) \leq h=j_{1}^{*}(Y, n+1) .
$$

Let $X^{\prime}=X-1 \mid X>0$ and similarly for $Y^{\prime}$. This implies that $Y^{\prime}<_{\mathrm{hr}} X^{\prime}$. By induction, $J_{n+1}^{*}(X)=\left(g, J_{n+1-g}^{*}\left(X^{\prime}\right)\right)$ is majorized by $\left(g, J_{n+1-g}^{*}\left(Y^{\prime}\right)\right)=\hat{J}_{n+1}(g, Y)$. But, from Lemma $2, J_{n+1}^{*}(Y)=\hat{J}_{n+1}(h, Y)$ majorizes $\hat{J}_{n+1}(g, Y)$. Hence the result follows.

Below is an example that shows that if $Y<_{\mathrm{hr}} X$ is weakened to $Y<_{\mathrm{st}} X$, then $J^{*}(Y)$ does not necessarily majorize $J^{*}(X)$. For that matter, in this example, $j_{1}^{*}(X)>j_{1}^{*}(Y)$. Assume that there are $n=3$ missiles. Let $u=0$ so that $c(j)=a(j)$. Let $c=(0.86,0.99,1.00)$. Let $\mathbb{P}(X=i)$ be $0.01,0.71$, and 0.28 for $i=1,2,3$, respectively, and let $\mathbb{P}(Y=i)$ be $0.71,0.01$, and 0.28 for $i=1,2,3$, respectively. Then it is easy to verify that $Y<_{\text {st }} X$, but it is not the case that $Y<_{\mathrm{hr}} X$. It turns out that $J_{3}^{*}(X)=(2,1,0)$ and $J_{3}^{*}(Y)=(1,1,1)$.

\section{The bomber problem}

Perhaps most interesting is Conjecture B in the bomber problem, because it has remained an open problem for the online, continuous-time $M(q, u)$ model for 45 years. It has been shown to be false for models different from $M(q, u)$ and discrete time. (See [10].) The only case where Conjecture B holds, according to a discrete-time setting discussed in Weber, is when there is an 
TABLE 2: Probability of surviving for $M(0.5,0.1,3.75)$ and $M(0.5,0,4.0)$.

\begin{tabular}{|c|c|c|c|c|c|c|c|c|c|c|c|c|c|c|c|c|}
\hline \multicolumn{8}{|c|}{$n=6$} & \multicolumn{9}{|c|}{$n=7$} \\
\hline$j_{1}$ & $j_{2}$ & $j_{3}$ & $j_{4}$ & $j_{5}$ & $j_{6}$ & $B(\cdot)$ & $B f(\cdot)$ & $j_{1}$ & $j_{2}$ & $j_{3}$ & $j_{4}$ & $j_{5}$ & $j 6$ & $j_{7}$ & $B(\cdot)$ & $B f(\cdot)$ \\
\hline 6 & 0 & 0 & 0 & 0 & 0 & 0.1055 & 0.0721 & 7 & 0 & 0 & 0 & 0 & 0 & 0 & 0.1063 & 0.0727 \\
\hline 5 & 1 & 0 & 0 & 0 & 0 & 0.1863 & 0.1420 & 6 & 1 & 0 & 0 & 0 & 0 & 0 & 0.1890 & 0.1442 \\
\hline 4 & 2 & 0 & 0 & 0 & 0 & 0.2208 & 0.1717 & 5 & 2 & 0 & 0 & 0 & 0 & 0 & 0.2274 & 0.1774 \\
\hline 4 & 1 & 1 & 0 & 0 & 0 & 0.2340 & 0.1832 & 5 & 1 & 1 & 0 & 0 & 0 & 0 & 0.2410 & 0.1893 \\
\hline 3 & 3 & 0 & 0 & 0 & 0 & 0.2264 & 0.1763 & 4 & 3 & 0 & 0 & 0 & 0 & 0 & 0.2408 & 0.1889 \\
\hline 3 & 2 & 1 & 0 & 0 & 0 & 0.2781 & 0.2244 & 4 & 2 & 1 & 0 & 0 & 0 & 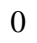 & 0.2957 & 0.2404 \\
\hline 3 & 1 & 1 & 1 & 0 & 0 & 0.2454 & 0.1923 & 4 & 1 & 1 & 1 & 0 & 0 & 0 & 0.2609 & 0.2061 \\
\hline 2 & 2 & 2 & 0 & 0 & 0 & 0.2736 & 0.2198 & 3 & 3 & 1 & 0 & 0 & 0 & 0 & 0.3071 & 0.2511 \\
\hline 2 & 2 & 1 & 1 & 0 & 0 & 0.2734 & 0.2198 & 3 & 2 & 2 & 0 & 0 & 0 & 0 & 0.3133 & 0.2564 \\
\hline 2 & 1 & 1 & 1 & 1 & 0 & 0.2233 & 0.1722 & 3 & 2 & 1 & 1 & 0 & 0 & 0 & 0.3137 & 0.2564 \\
\hline 1 & 1 & 1 & 1 & 1 & 1 & 0.1606 & 0.1164 & 3 & 1 & 1 & 1 & 1 & 0 & 0 & 0.2557 & 0.2009 \\
\hline & & & & & & & & 2 & 2 & 2 & 1 & 0 & 0 & 0 & 0.3175 & 0.2610 \\
\hline & & & & & & & & 2 & 2 & 1 & 1 & 1 & 0 & 0 & 0.2866 & 0.2308 \\
\hline & & & & & & & & 2 & 1 & 1 & 1 & 1 & 1 & 0 & 0.2263 & 0.1746 \\
\hline & & & & & & & & 1 & 1 & 1 & 1 & 1 & 1 & 1 & 0.1613 & 0.1169 \\
\hline
\end{tabular}

enemy plane at each time instant. This corresponds to $X$ being identically an integer, $m$, in our setting. For this case, clearly the online and offline situations are identical, and Conjecture B holds for $c(j) \log$-concave.

Assertion 2. For the offline bomber problem, Conjecture $B$ is false even for an $M(q, u, t)$ model.

Specifically, we consider $t=3.75, u=0.1$ and $q=0.5$. Table 2 lists the probability of surviving for the 11 possible configurations for $n=6$ and the 15 possible configurations for $n=7$. As can be seen from Table $2, J_{6}^{*}=(3,2,1,0,0,0)$ is the optimal allocation for $n=6$ and $J_{7}^{*}=(2,2,2,1,0,0,0)$ is the optimal allocation for $n=7$. Hence, the number of missiles used at the outset for $n=7$ is less than the number of missiles used at the outset for $n=6$. We also provide a counterexample in the table for the frail fighter problem (i.e. $u=0$ ). The optimal allocations are the same as in the above example. We use $t=4.0$ and $q=0.5$, and indicate the results as $B f$ in Table 2 .

We now show that Conjecture $\mathrm{C}$ holds for the bomber problem. Before stating and proving the theorem note that, from (5),

$$
B\left(X, J_{n}^{*}\right)=\mathbb{P}(X=0)+c\left(j_{1}^{*}\right)\left[\mathbb{P}(X=1)+R_{B}\left(X^{\prime}, n-j_{1}^{*}\right)\right],
$$

where $R_{B}\left(X^{\prime}, n-j\right)$ has a parallel interpretation to that of $R_{F}\left(X^{\prime}, n-j\right)$.

Theorem 4. Assume that the $c(j)$ sequence is log-concave. Then for any $X$, Conjecture $C$ holds for the offline bomber problem.

Proof. We use the same notation as in the proof of Theorem 2. Again, assume that the claim is false, i.e. that $j_{1}^{*}(n+1)>j_{1}^{*}(n)+1$. Since $j$ is optimal for $n$, it is preferred to $h-1$ for $n$, and since $h$ is optimal for $n+1$, it is preferred to $j+1$ for $n+1$. Using (14), this yields

$$
c(j)\left[\mathbb{P}(X=1)+R_{B}\left(X^{\prime}, n-j\right)\right] \geq c(h-1)\left[\mathbb{P}(X=1)+R_{B}\left(X^{\prime}, n+1-h\right)\right],
$$


i.e.

$$
\frac{c(j)}{c(h-1)} \geq \frac{\mathbb{P}(X=1)+R_{B}\left(X^{\prime}, n+1-h\right)}{\mathbb{P}(X=1)+R_{B}\left(X^{\prime}, n-j\right)}
$$

and

$$
c(h)\left[\mathbb{P}(X=1)+R_{B}\left(X^{\prime}, n+1-h\right)\right]>c(j+1)\left[\mathbb{P}(X=1)+R_{B}\left(X^{\prime}, n-j\right)\right],
$$

i.e.

$$
\frac{c(j+1)}{c(h)}<\frac{\mathbb{P}(X=1)+R_{B}\left(X^{\prime}, n+1-h\right)}{\mathbb{P}(X=1)+R_{B}\left(X^{\prime}, n-j\right)} .
$$

Under $j_{1}^{*}(n+1)>j_{1}^{*}(n)+1$, the inequality is strict by the convention of choosing $J^{*}$ with minimum first entry in the case of ties. By log-concavity,

$$
\frac{c(j+1)}{c(h)} \geq \frac{c(j)}{c(h-1)} ;
$$

thus, (15) and (16) contradict each other, and $j_{1}^{*}(n+1)>j_{1}^{*}(n)+1$ is false.

We now consider Conjecture A for the bomber problem.

Theorem 5. If $Y<\operatorname{lr} X$ and the c-sequence is log-concave and nondecreasing, then $j_{1}^{*}(Y) \geq$ $j_{1}^{*}(X)$.

We omit the details of the proof as it follows closely the proof for the fighter problem. The analogue to Lemma 2 requires Conjecture $C$. Conjecture $C$ follows from Theorem 4 . The only other change is to (17) below, where $\mathbb{P}(X \geq i)$ now becomes $\mathbb{P}(X=i)$ and $v^{\prime}$ becomes $\prod_{l=k+1}^{i} c\left(j_{i}\right)$. These modifications are only used in claiming that $v^{\prime}$ depends solely on the last $n-k$ observations. The analogue to Lemma 3 is to show that if $J$ majorizes $J^{\prime}$ then $S_{n}(J) \leq S_{n}\left(J^{\prime}\right)$. The algorithm in the appendix shows this (as does [10]). We can transform $J$ to $J^{\prime}$ through a series of steps that adjusts an allocation by increasing the number of missiles by one for the $k$ th potential encounter and reducing the number of missiles by one for the potential $k^{\prime}$ th encounter, where $k<k^{\prime}$. The above step does not decrease the probability of surviving through the $n$th encounter since $c(g+1) c(h-1) \geq c(g) c(h)$ for any $g<h$ by log-concavity.

The only difference is in Lemma 4 where we explicitly use $Y<_{\mathrm{hr}} X$ in the fighter problem, which implies that $\mathbb{P}(X \geq k) / \mathbb{P}(Y \geq k)$ is nondecreasing in $k$. To adapt Lemma 4 to the bomber problem we therefore need $\mathbb{P}(X=k) / \mathbb{P}(Y=k)$ to be nondecreasing in $k$. This is guaranteed by the likelihood ratio order. Then Conjecture A holds.

Corollary 2. For the bomber problem, if $Y<\operatorname{lr} X, J^{*}(Y)$ majorizes $J^{*}(X)$.

Below is an example that shows that if $Y<_{\operatorname{lr}} X$ is weakened to $Y<_{\mathrm{hr}} X$, then $J^{*}(Y)$ does not necessarily majorize $J^{*}(X)$. For that matter, in this example, $j_{1}^{*}(X)>j_{1}^{*}(Y)$. Assume that there are $n=4$ missiles. Let $c=(0.05,0.10,0.14,0.18)$. Let $\mathbb{P}(X=i)$ be $0.12,0.03$, and 0.85 , respectively, and let $\mathbb{P}(Y=i)$ be $0.13,0.86$, and 0.01 for $i=1,2,3$, respectively. Then it is easy to verify that $Y<_{\mathrm{hr}} X$, but it is not the case that $Y<_{\mathrm{lr}} X$. It turns out that $J^{*}(X)=(4,0,0)$ and $J^{*}(Y)=(3,1,0)$.

\section{Relationship between online and offline problems}

It is clear that the online versions of the fighter problem and bomber problem will do at least as well as their corresponding offline versions. The issue is how much better does one 
do if the allocation can be made dynamically so that the times of the encounters (which relates to the distributions for the number of remaining encounters) can be exploited. This is studied numerically by considering the $M(q, u, t)$ models for $q=0.1,0.5,0.9$ and $u=0,0.1,0.5,0.9$, where the numbers of missiles are $n=3,5,10,20$ and $t=1,2, \ldots, 20$.

We first consider the fighter problem. The offline version is implemented by considering all possible allocations that satisfy $j_{1} \geq j_{2} \geq \cdots \geq j_{n}$. There are only 627 possible allocations when $n=20$. Note that in Section 24.2.1 of [1] there is an asymptotic formula, $(1 / 4 n \sqrt{3}) \mathrm{e}^{\pi \sqrt{2 / 3} / \sqrt{n}}$, for the number of integrand summands of $n$ without regard to order. This asymptotic formula yields 692.4 when $n=20$. In order to obtain the maximum expected number of enemy planes that are shot down dynamically, we consider

$$
F^{*}(t, n)=\max _{1 \leq j \leq n}\left[a(j)+c(j) \int_{s=0}^{t} F^{*}(s, n-j) \mathrm{e}^{s-t} \mathrm{~d} s\right],
$$

where $F^{*}(t, n)$ denotes the expected number of enemy planes shot down, when meeting an enemy at time $t$, and $n$ missiles are at hand, with $F^{*}(t, 0)=0$. For fixed $n$, in order to evaluate $F^{*}(t, n)$ by recursion, we approximate the above integral by a sum with very small increments. Since the first encounter is not necessarily at $t$, the optimal objective is

$$
\int_{0}^{t} F^{*}(s, n) \mathrm{e}^{s-t} \mathrm{~d} s .
$$

For fixed $n$, the objective functions for the offline and online versions increase as a function of $t$. We consider $0<t<20$. The advantage of the online solution is small when $t$ is small as a large number of missiles will be used at the first encounter. In the limit as $t$ goes to $\infty$ there is no advantage, as, with probability tending to 1 , there will be more than $n$ encounters, and the optimal allocation for the online and offline situations will be the same. It is when $t$ is of moderate size relative to $n$ that the advantage is greatest for the online version. For example, if $u=0.5$ and $q=0.5$, the maximum gain of $2.8 \%$ of the online version, relative to the offline version occurs when $t=0.639$ for $n=3$. The respective maximum gains and times are: $2.2 \%$ at $t=2.161$ for $n=5 ; 1.3 \%$ at $t=1.677$ for $n=10 ; 1.3 \%$ at $t=4.988$ for $n=20$.

In looking at Figure 1, where the rows correspond to increasing values of $u$ and the columns correspond to increasing values of $q$, it appears that, as $u$ increases, the relative gain from the online to offline versions increases. On the other hand, when $q=0.5$, its middle value is where this ratio appears largest. But, the main conclusion is that there is little gain in using the dynamic approach rather than determining the spending policy ahead. In the extreme in all cases studied, the maximum advantage of the dynamic approach is about $3 \%$.

The numerical results for the bomber problem are obtained in a similar manner to those for the fighter problem (see Figure 2). For the offline version, the best allocation is obtained by complete enumeration over the feasible set of allocations. There exist recursive relations also for the offline problem, but these seem to have little computational advantage. Fortunately, the size of the feasible set is not too large even when $n$ reaches 50. The online solution is found recursively through the relationship

$$
B^{*}(t, n)=\max _{1 \leq j \leq n} c(j) \int_{s=0}^{t} B^{*}(s, n-j) \mathrm{e}^{s-t} \mathrm{~d} s,
$$

where $B^{*}(t, n)$ is the optimal probability of reaching the target $t$, in the online situation, when meeting an enemy at time $t, n$ missiles are available, and $B^{*}(t, 0)=0$. Since the time of the 

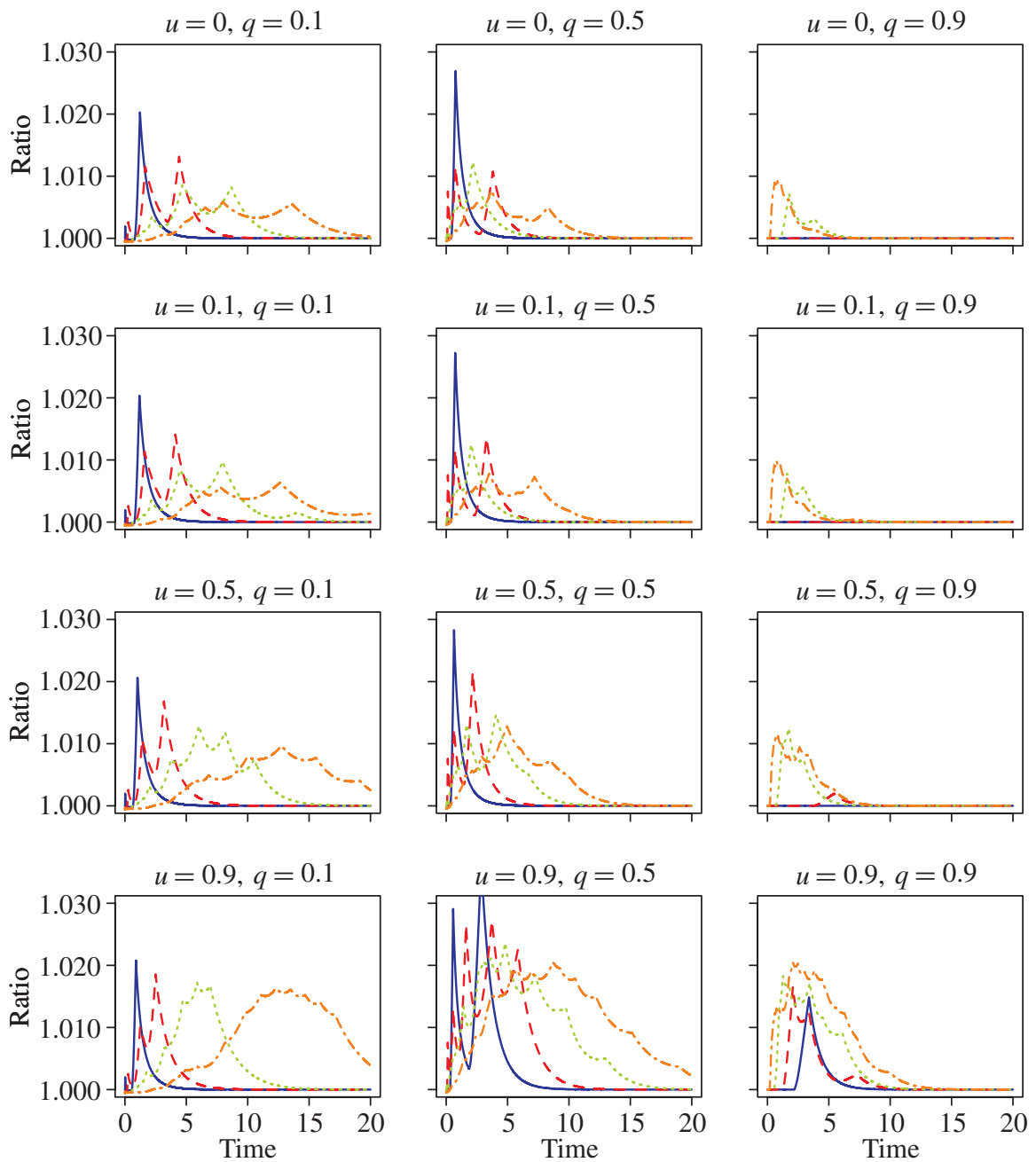

Figure 1: Ratio of online to offline for fighter $M(q, u, t)$ models, for $n=3$ (solid blue lines), $n=5$ (dashed red lines), $n=10$ (dotted green lines), and $n=20$ (dash-dot orange lines).

first encounter is not necessarily $t$, the value of the optimal objective is

$$
\int_{0}^{t} B^{*}(s, n) \mathrm{e}^{s-t} \mathrm{~d} s+\mathrm{e}^{-t} .
$$

The probability of reaching the target is much greater in the online version relative to the offline version. The relative advantage in using an online policy is much higher for the bomber than for the fighter, and in the numerical examples considered reaches $30 \%$ in some cases. The relative gain appears to decrease with increasing values of $u$. So it is the frail bomber who gains most by being able to observe the times of the encounters before deciding on the number of missiles to be used. As in the fighter problem, the gain is greatest when $q$ is in the middle of the range. Although this is not uniform across the cases, the greater the number of missiles that are available at the outset, $n$, the more value there is in the online solution relative to the offline solution. 

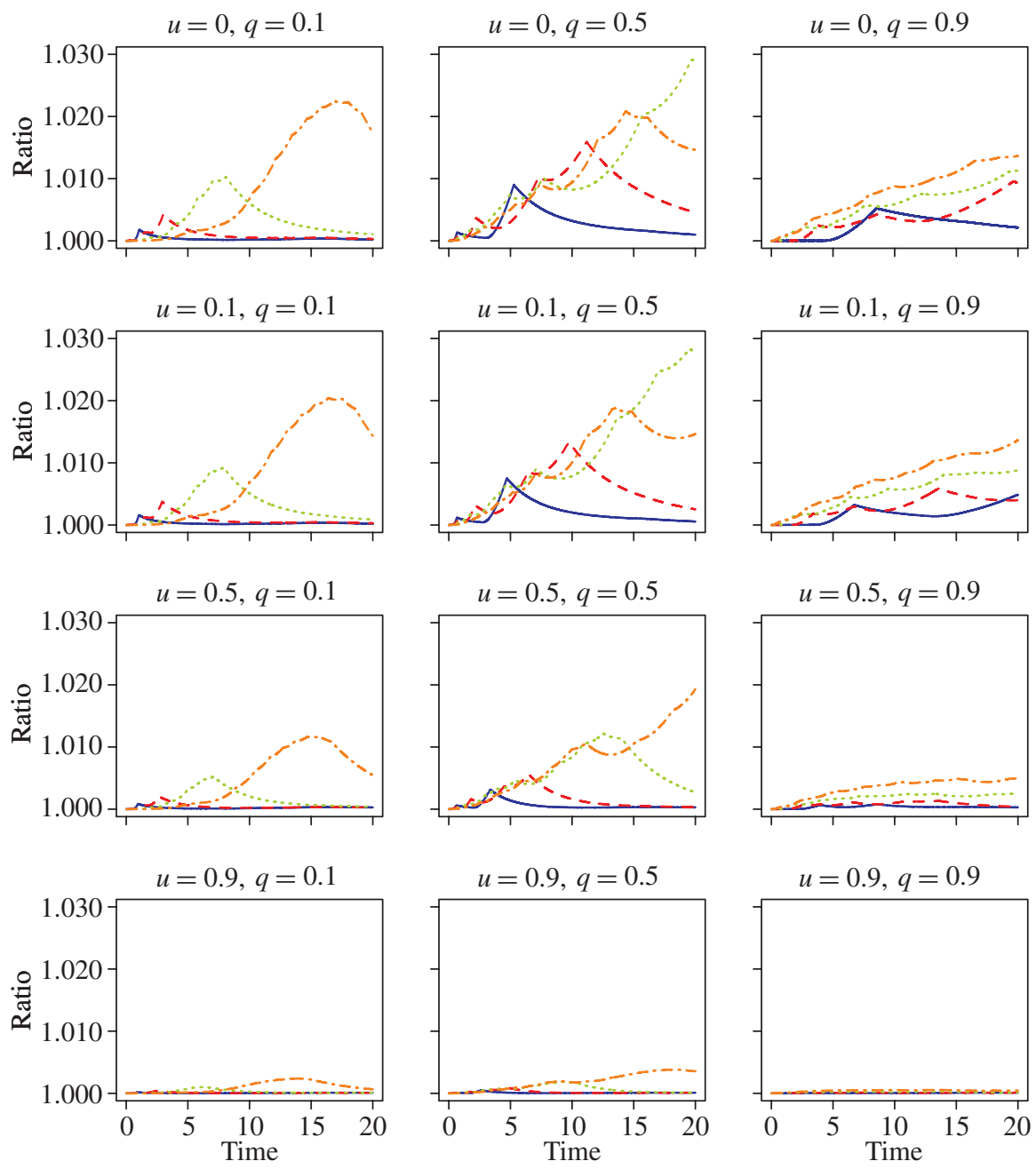

Figure 2: Ratio of online to offline for bomber $M(q, u, t)$ models, for $n=3$ (solid blue lines), $n=5$ (dashed red lines), $n=10$ (dotted green lines), and $n=20$ (dash-dot orange lines).

It is not difficult to see that, for fixed $n$, as $t$ tends to $\infty$, the ratio tends to 1 , both for the bomber problem and fighter problem. Similarly, for fixed $t$, as $n$ tends to $\infty$, these ratios again tend to 1 . Thus, the interesting cases are when $n$ and $t$ are of 'comparable' size.

\section{Appendix A. Proofs of Lemmas 2, 3, and 4}

Proof of Lemma 2. Let $\hat{J}(h, X)=J=\left(j_{1}, \ldots, j_{n}\right)$ and $\hat{J}(g, X)=J^{\prime}=\left(j_{1}^{\prime}, \ldots, j_{n}^{\prime}\right)$. Since in our case $j_{1}^{\prime}<j_{1}$, it follows from Conjecture $\mathrm{C}$ that there exists a $k$ with $\mathbb{P}(X \geq k)>0$ such that $\sum_{i=1}^{k-1} j_{i}^{\prime}<\sum_{i=1}^{k-1} j_{i}$ and $\sum_{i=1}^{k} j_{i}=\sum_{i=1}^{k} j_{i}^{\prime}$. Of course, if $k=n$, the length of the vectors, we are done. Otherwise, we show that $j_{i}=j_{i}^{\prime}$ for $i=k+1, \ldots, n$, that is, once the two sequences catch up at some $k$, the optimal allocation from there on must be the same. 
Consider the contribution to $F(X, J)$ from the last $n-k$ terms,

$$
s_{k}(J) \sum_{i=k+1}^{n} \mathbb{P}(X \geq i) v_{i}^{\prime}(J),
$$

where

$$
v_{i}^{\prime}(J)= \begin{cases}a\left(j_{i}\right) & \text { if } i=k+1, \\ \prod_{l=k+1}^{i-1} c\left(j_{l}\right) a\left(j_{i}\right) & \text { if } i>k+1 .\end{cases}
$$

Note that $s_{k}(J)$ depends on the first $k$ entries of $J$ and the terms in the sum in (17) depend only on the last $n-k$ entries. If the sum above is maximized at $\left(j_{k+1}^{*}, \ldots, j_{n}^{*}\right)$ then this would be the optimal way to complete the allocation regardless of the first $k$ entries.

Proof of Lemma 3. Let $J=\hat{J}(h, X)$ and $J^{\prime}=\hat{J}(g, X)$ with $g<h$. From Lemma 2 , $J$ majorizes $J^{\prime}$. Let $m=\operatorname{argmax}_{k}\left\{j_{k}>0\right\}$ and $\tilde{m}=\operatorname{argmax}_{k}\left\{j_{k}^{\prime}>0\right\}$. Clearly, $m \leq \tilde{m}$.

If $m<\tilde{m}$ then $v_{\tilde{m}}(J)=0$ and we are done.

If $m=\tilde{m}$ then $v_{m}=v_{m}(J)=s_{m-1}(J) a\left(j_{m}\right)$, and similarly for $v_{m}^{\prime}$. Note that $j_{m} \leq j_{m}^{\prime}$. If $j_{m}=j_{m}^{\prime}$ then we are done, since $s_{m-1}(J) \leq s_{m-1}\left(J^{\prime}\right)$ from the algorithm below. We therefore need to show that if $j_{m}<j_{m}^{\prime}$ then $v_{m}=s_{m-1}(J) a\left(j_{m}\right) \leq v_{m}^{\prime}=s_{m-1}\left(J^{\prime}\right) a\left(j_{m}^{\prime}\right)$.

Consider the allocation $J^{\prime \prime}$ defined by $j_{k}^{\prime \prime}=j_{k}^{\prime}$ for $k<m-1, j_{m-1}^{\prime \prime}=j_{m-1}^{\prime}+j_{m}^{\prime}-j_{m}$, and $j_{m}^{\prime \prime}=j_{m}$. The contribution to the objective function of the potential $(m-1)$ th encounter is at least as great for allocation $J^{\prime \prime}$ as it is for allocation $J^{\prime}$ and the contribution to all prior potential encounters are the same. Since $J^{\prime}$ is the best allocation that uses $g$ missiles on the first encounter, this implies that the contribution of the $m$ th potential encounter is greater for allocation $J^{\prime}$ than for $J^{\prime \prime}$. Furthermore, $J$ majorizes $J^{\prime \prime}$; hence, $s_{m-1}\left(J^{\prime \prime}\right) \geq s_{m-1}(J)$. This implies that

$$
v_{m}=s_{m-1}(J) a\left(j_{m}\right) \leq s_{m-1}\left(J^{\prime \prime}\right) a\left(j_{m}\right) \leq v_{m}^{\prime}=s_{m-1}\left(J^{\prime}\right) a\left(j_{m}^{\prime}\right),
$$

as desired.

The result that if $J$ majorizes $J^{\prime}$ then $s_{m}(J) \leq s_{m}\left(J^{\prime}\right)$ is given in [10]. It follows more directly from the algorithm below that transforms $J$ to $J^{\prime}$ without decreasing the probability of survival through $m$ potential encounters.

\section{Algorithm.}

Step 0. Let $J^{\prime \prime}=J^{\prime}$.

Step 1. Let $k=\operatorname{argmax}_{1 \leq l \leq m}\left\{j_{l}^{\prime \prime}<j_{l}\right\}$.

Let $i=\operatorname{argmax}_{1 \leq l \leq k}\left\{j_{l}^{\prime \prime}>j_{l}\right\}$.

If $k$ exists, so will $i$ as $\sum_{l=1}^{k} j_{l}^{\prime \prime} \leq \sum_{l=1}^{k} j_{l}^{\prime}$. If $k$ does not exist then $J^{\prime \prime}=J$ and stop.

Step 2. Let $j_{k}^{\prime \prime} \leftarrow j_{k}^{\prime \prime}+1$.

$j_{i}^{\prime \prime} \leftarrow j_{i}^{\prime \prime}-1$.

Go to step 1.

Note that, since $\sum_{i=1}^{k} j_{i}^{\prime \prime} \leq \sum_{i=1}^{k} j_{i}^{\prime}, j_{k-1}^{\prime \prime} \geq j_{k-1} \geq j_{k} \geq j_{k}^{\prime \prime}$. Hence, $j_{k-1}^{\prime \prime}-j_{k}^{\prime \prime} \geq 1$, so we can increase $j_{k}^{\prime \prime}$. Similarly, $j_{i}^{\prime \prime}>j_{i} \geq j_{i+1} \geq j_{i+1}^{\prime \prime}$, so $j_{i}^{\prime \prime}$ can be decreased by 1 . The reason $s_{k}\left(J^{\prime \prime}\right)$ does not decrease from this step follows from $c(j-1) c(h+1) \geq c(j) c(h)$ for $j>h$. 
Proof of Lemma 4. Since $v_{1}^{\prime} \leq v_{1}$ and $v_{n}^{\prime} \geq v_{n}$, there exist $H$ subsets of contiguous indices such that $v_{i}^{\prime}-v_{i}$ goes from nonpositive to nonnegative. Denote these $H$ subsets of $\{1, \ldots, n\}$ as $\left[b_{h}, e_{h}\right], h=1, \ldots, H$, with $b_{1}=1, e_{H}=n$, and $b_{h+1}=e_{h}+1$. Then there exist indices $m_{h}$ such that $v_{j}^{\prime} \leq v_{j}$ for $b_{h} \leq j \leq m_{h}$ and $v_{j}^{\prime} \geq v_{j}$ for $m_{h} \leq j \leq e_{h}$. Hence,

$$
\begin{aligned}
\sum_{k=1}^{n} \mathbb{P}(X \geq k)\left(v_{k}^{\prime}-v_{k}\right) & =\sum_{h=1}^{H} \sum_{j=b_{h}}^{e_{h}} \mathbb{P}(X \geq j)\left(v_{j}^{\prime}-v_{j}\right) \\
& =\sum_{h=1}^{H} \sum_{j=b_{h}}^{e_{h}} \frac{\mathbb{P}(X \geq j)}{\mathbb{P}(Y \geq j)} \mathbb{P}(Y \geq j)\left(v_{j}^{\prime}-v_{j}\right) \\
& \geq \sum_{h=1}^{H} \frac{\mathbb{P}\left(X \geq m_{h}\right)}{\mathbb{P}\left(Y \geq m_{h}\right)} \sum_{j=b_{h}}^{e_{h}} \mathbb{P}(Y \geq j)\left(v_{j}^{\prime}-v_{j}\right) \\
& \geq \min _{\{1 \leq h \leq H\}} \frac{\mathbb{P}\left(X \geq m_{h}\right)}{\mathbb{P}\left(Y \geq m_{h}\right)} \sum_{h=1}^{H} \sum_{j=1}^{e_{h}} \mathbb{P}(Y \geq j)\left(v_{j}^{\prime}-v_{j}\right) \\
& \geq 0 .
\end{aligned}
$$

Note that the first inequality is the result of $Y<\mathrm{hr} X$ and uses (1).

\section{References}

[1] Abramowitz, M. And Stegun, I. A. (1972). Handbook of Mathematical Functions with Formulas, Graphs, and Mathematical Tables. US Government Printing Office, Washington, DC.

[2] Bartroff, J. And SAmuel-CAHn, E. (2011). The fighter problem: optimal allocation of a discrete commodity. Adv. Appl. Prob. 43, 121-130.

[3] Bartroff, J., Goldstein, L., Rinott, Y. and Samuel-Cahn, E. (2010). On optimal allocation of a continuous resource using an iterative approach and total positivity. Adv. Appl. Prob. 42, 795-815.

[4] Klinger, A. And Brown, T. A. (1968). Allocating unreliable units to random demands. In Stochastic Optimization and Control (Madison, Wisconsin, 1967), ed. H. F. Karreman, John Wiley, New York, pp. 173-209.

[5] Samuel, E. (1970). On some problems in operations research. J. Appl. Prob. 7, 157-164.

[6] Shaked, M. and Shanthikumar, J. G. (2007). Stochastic Orders. Springer, New York.

[7] Shepp, L. A., Simons, G. And Yao, Y.-C. (1991). On a problem of ammunition rationing. Adv. Appl. Prob. 23, 624-641.

[8] Simons, G. And Yao, Y.-C. (1990). Some results on the bomber problem. Adv. Appl. Prob. 22, $412-432$.

[9] Weber, R. R. (1985). A problem in ammunition rationing. In Stochastic Dynamic Optimization and Applications in Scheduling and Related Areas, University of Passau, p. 148.

[10] Weber, R. (2011). ABCs of the bomber problem and its relatives. Ann. Operat. Res. 22pp. (forthcoming). 